\title{
WHAT HAVE WE LEARNT FROM
}
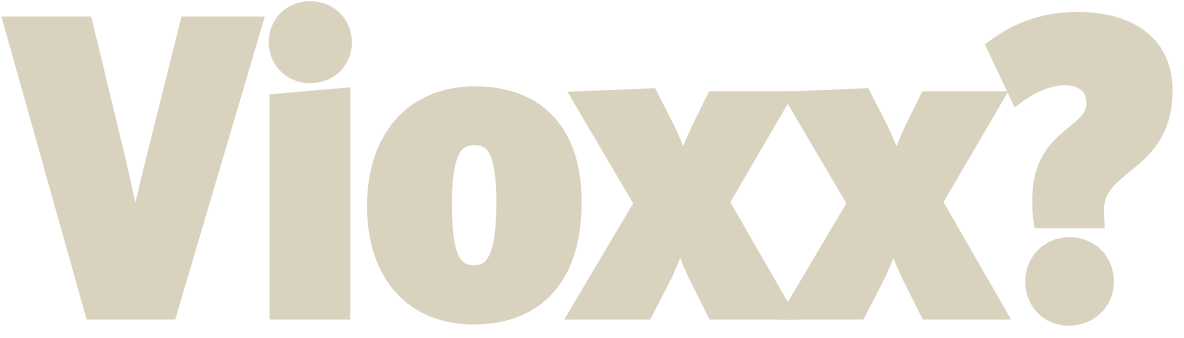

\section{In October UK patients who had cardiovascular events while taking rofecoxib lost the right to fight Merck in the US for compensation. But researchers and journals can still benefit from this case if they learn from the mistakes,} write Harlan Krumholz and colleagues

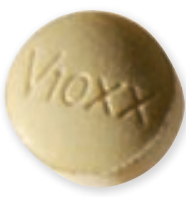

Rofecoxib (Vioxx) was introduced by Merck in 1999 as an effective, safer alternative to non-steroidal anti-inflammatory drugs for the treatment of pain associated with osteoarthritis. It was subsequently found to increase the risk of cardiovascular disease and withdrawn from the worldwide market. Merck now faces legal claims from nearly 30000 people who had cardiovascular events while taking the drug. ${ }^{1}$ The company has stated that it will fight each case, denying liability. ${ }^{2}$ Our recent participation in litigation at the request of plaintiffs provided a unique opportunity to thoroughly examine and reflect on much of the accumulated court documents, research, and other evidence. This story offers important lessons about how best to promote constructive collaboration between academic medicine and industry.

\section{Early suspicion of cardiovascular risk}

Since the early development of rofecoxib, some scientists at Merck were concerned that the drug might adversely affect the cardiovascular system by altering the ratio of prostacyclin to thromboxane, which act in opposition, balancing blood flow and clotting. ${ }^{\text {w1 }}$ A study sponsored by Merck during 1996-7 reported that rofecoxib reduced urinary metabolites of prostacyclin in healthy volunteers by about half. ${ }^{\text {w2 }}$ In internal emails made public through litigation, ${ }^{3}$ Merck officials sought to soften the academic authors' interpretation that cyclo-oxygenase- 2 (COX 2) inhibition within the vascular endothelium may increase the propensity for thrombus formation, the basis of what became known as the FitzGerald hypothesis. ${ }^{\text {w3 }}$ The academic authors changed the manuscript at Merck's request-for example, they changed "systemic biosynthesis of prostacyclin ... was decreased by [rofecoxib]" to "Cox-2 may play a role in the systematic biosynthesis of prostacyclin." 3 w2 To the authors' credit, they continued to investigate the effects of COX 2 inhibition and ultimately provided much of the basic science knowledge that clarified the pathways by which rofecoxib probably leads to cardiovascular events. ${ }^{\text {w-w7 }}$

However, despite Merck's knowledge that rofecoxib might increase thrombus formation, none of the intervention studies that constituted its new drug application to the Food and Drug Administration in 1998 were designed to evaluate cardiovascular risk. The nine studies were generally small, had short treatment periods, enrolled patients at low risk of cardiovascular disease, and did not have a standardised procedure to collect and adjudicate cardiovascular outcomes. ${ }^{4}$ Moreover, Merck seemingly pooled data from these studies and others for analysis of cardiovascular risks, despite FDA concern, ${ }^{5}$ and disseminated the results to promote the drug's cardiovascular safety to doctors in its "cardiovascular card," 67 a marketing device cited by US Congressman Henry Waxman for falsely minimising cardiovascular risks ${ }^{8}$ and never approved by the FDA.

\section{The VIGOR study}

In January 1999, Merck launched its largest study yet of rofecoxib, the Vioxx gastrointestinal outcomes research (VIGOR) study. The study was intended to expand the drug's approved indications by showing that it would have fewer gastrointestinal side effects than naproxen for the treatment of rheumatoid arthritis. The study of over 8000 patients was initiated without a standard operating procedure for collecting information on cardiovascular events and without a cardiologist on the data safety monitoring board. Data safety monitoring boards are independent committees whose purpose is to monitor the results of an ongoing trial to ensure the safety of trial participants. ${ }^{\text {w8 }}$ The study was designed to continue until a predetermined number of confirmed uncomplicated or complicated gastric perforations, ulcers, or bleeds had occurred.

The first non-endpoint safety analysis was presented to the safety board in November 1999 , at which time a $79 \%$ greater risk of 
$J A M A$ that raised questions about the safety of rofecoxib and the validity of the naproxen hypothesis, ${ }^{\text {w12 }}$ few academic researchers publicly questioned the company before its voluntary withdrawal of the drug. Moreover, Merck selectively targeted doctors who raised questions about the drug, going so far as pressurising some of them through department chairs. ${ }^{16}$

\section{Short and long term use}

For several years, Merck continued to investigate other indications for rofecoxib and conducted additional trials. The increased cardiovascular risk compared with placebo was reported in a 2004 analysis of the adenomatous polyp prevention on Vioxx (APPROVe) study, ${ }^{\text {w13 }}$ which led to the drug's withdrawal. The financial implications were immense not only because of loss of revenue but also because of expected litigation. The key question was when the risk became manifest. If short term use was not associated with increased cardiovascular risk, Merck's liability would potentially be drastically reduced.

The APPROVe authors, five of whom were Merck employees and the remainder of whom received consulting fees from Merck, asserted that the increased risk became apparent only after 18 months of use. ${ }^{\text {w13 }}$ This conclusion was based on an analysis that was not prespecified and a flawed methodological approach. Merck subsequently admit-

\section{Merck selectively targeted doctors who raised questions about the drug, going so far as pressurising some of them through department chairs}

of concern"w9 w10 and a correction ${ }^{\mathrm{w} 14}$ and publishing a methodological paper ${ }^{\mathrm{w} 15}$ and other related comments and editorials. ${ }^{\text {w16-w24 }}$ But other academic medical journals also played important parts. In 2001, Circulation published a pooled analysis of 23 phase IIb$\mathrm{V}$ studies examining the association between rofecoxib and cardiovascular risk. The paper had no editorial commentary or critique, ${ }^{\text {w25 }}$ even though the study was coordinated internally at Merck, the results highly favoured the safety of rofecoxib, and five of the seven authors were Merck employees (the two academic authors acknowledged being paid consultants to Merck). Moreover, in internal emails made public through litigation, even an executive scientist at Merck criticised the analysis, stating: "The data appears to have been interpreted to support a preconceived hypothesis rather than critically reviewing the data to generate hypotheses." 17

The Annals of Internal Medicine published the assessment of differences between Vioxx and naproxen to ascertain gastrointestinal tolerability and effectiveness (ADVANTAGE) study. ${ }^{\text {w26 }}$ It later learnt that article was written by Merck without accreditation, ${ }^{\text {w27 }}$ ${ }^{\text {w } 28}$ contained errors in the presentation of cardiovascular events with rofecoxib (minimising cardiovascular risk), and was conducted for marketing purposes, a so called seeding trial. The journal was quick to condemn ghostwriting ${ }^{\text {w29 }}$ and a full correction of the errors was published recently ${ }^{\text {w30 }}$ after Merck scientists ted that it had incorrectly described the statistical approach, and the New England Journal of Medicine issued a correction indicating that statements regarding an increase in risk after 18 months should be removed from the article. ${ }^{\mathrm{w} 14}$ Again, mistakes that favoured the company, with colossal economic implications, made it through the journal peer review process to the profession and the public.

\section{Medical journals}

The New England Journal of Medicine has had a prominent role in the story. It published the VIGOR and APPROVe studies, responding to their inaccuracies with "an expression provided an initial, but incorrect explanation. ${ }^{\text {w31 }}$ Many other journals have published articles with results favourable to rofecoxib that court documents have shown to be ghostwritten by scientific writing companies hired by Merck. ${ }^{\text {w32-w36 }}$

\section{Promoting constructive collaboration}

The rofecoxib case is bad news for industry, academics, journals, and the public. Merck was once one of the US's most publicly admired companies, ${ }^{\text {w37 }}$ and its behaviour may not be different from that of others in the pharmaceutical or biotechnology industry. Journalists have questioned the ethics of industry and academic researchers. ${ }^{18-20}$ And yet, there is hardly a sense of outrage in the profession about the events that transpired.

Defenders of Merck may say that we do not know how rofecoxib's cardiovascular risk compares with that of other COX 2 inhibitors or traditional non-steroidal antiinflammatory drugs. But the proper place of these drugs in the medical armamentarium is beside the point. With billions of dollars at stake, Merck conducted the trials, stored and analysed the data internally, paid academic researchers as consultants to the investigative teams and the safety monitoring boards, and maintained heavy involvement in the writing and presentation of findings. The journals published the studies, and the academic community accepted the findings without expressing much concern. Nearly 107 million prescriptions for rofecoxib were dispensed in the US between 1999 and September $2004,{ }^{21}$ when the drug was withdrawn from the market, and none of the people picking up those prescriptions had the opportunity to consider the true balance of its risks and benefits.

What should we do going forward? Academic medicine, industry, medical journals, and government agencies need to come together to define a set of principles by which we can restore faith in collaborations on new treatments that can improve patient care. We might consider adopting some new approaches. Academics engaged in industry designed and sponsored studies should insist that the data are stored on an academic site, analysed by non-company investigators, and eventually made accessible to the public for scrutiny. Several early, large clinical trials of rofecoxib were not published in the academic literature for years after Merck made them available to the FDA, ${ }^{22}$ preventing independent investigators from accurately determining its cardiovascular risk using meta-analysis. In addition, independent audits should be conducted to ensure that companies follow a standardised, prespecified protocol.

Independent data and safety monitoring boards should be mandated and their governance should not be under the control of the company. Industry should not be allowed to select who 
serves on these boards or allowed to compensate members after their service.

In considering articles for publications, journals should understand that studies with immense financial implications require a higher level of scrutiny than others, especially when the study is conducted by the company with the financial stake. Journals should be prepared to go beyond the usual high quality review, paying particular attention to the pos-

In considering articles
for publications,
journals should
understand that
studies with immense
financial implications
require a higher level
of scrutiny than others

208088, New Haven, CT 06520-8088, USA

Joseph S Ross is instructor, Department of Geriatrics and Adult Development, Mount Sinai School of Medicine, New York, USA

Amos $\mathrm{H}$ Presler is research associate, Never Again Consulting, Attleboro, MA, USA

David S Egilman is clinical associate professor, Department of Bio Med Community Health, Brown University, Providence, RI, USA

Correspondence to: HM Krumholz harlan.krumholz@yale.edu

Contributors and sources: HMK's research is focused on determining optimal clinical strategies and identifying opportunities for sibility of bias. Articles should be accompanied by editorials by people without financial conflicts of interest. Moreover, ghostwriting constitutes a false statement of authorship or a false attribution of authorship, and academic researchers who sign off or "edit" original publications or reviews written by industry should be penalised unless there is full disclosure of the authorship, such as: "Representatives from $X Y Z$ drafted the manuscript; the authors were responsible for critical revisions of the manuscript for important intellectual content."

Even the best oversight cannot always detect mistakes. When journals discover that information has been withheld or that results are incorrect, they need to rapidly disseminate that information and ensure that any web search that identifies the errant manuscript also identifies the correction. Authors should sign agreements that they will notify journals if such information becomes available or face being blacklisted by the journal.

Our system depends on putting patients' interests first. Collaborations between academics, practising doctors, industry, and journals are essential in advancing knowledge and improving the care of patients. Trust is a necessary element of this partnership, but the recent events have made it necessary to institute proper systems that protect the interests of patients. A renewed commitment by all those involved and the institution of these systems are the only way to extract something positive from this unfortunate affair. improvement in the prevention, treatment, and outcome of cardiovascular disease with emphasis on older populations. He was responsible for the concept and writing of this article and is its guarantor. JSR has studied and reported on conflict of interest in medicine. He participated in the concept and design of this article and revised it for critical content. AHP has research interests in the history of public health and law, including pharmaceutical research and marketing. He consults on this topic at the request of plaintiffs in the Vioxx litigation. He contributed document research and interpretation to this article and participated in its revision. DSE has studied and reported corporate corruption of science. He contributed to the development of this article and revised the work for critical content. This article arose from access to Merck documents as a result of tort litigation. Competing interests: HMK has research contracts with the American College of Cardiology and the Colorado Foundation for Medical Care; serves on the advisory boards of Amgen, Alere, and UnitedHealthcare; is a subject expert for VHA; and is editor in chief of Journal Watch Cardiology. All authors have been consultants at the request of plaintiffs for recent suits against Merck related to rofecoxib.

1 United States Securities and Exchange Commission. Form 10-Q: quarterly report pursuant to section 13 or 15(d) of the Securities Exchange Act of 1934 (Note 7 to consolidated financial statements). Merck, 2006; http://phx.corporate-ir.net/phoenix.zhtml?c=73184\& p=irolSECText\&TEXT=aHROcDovL2NjYm4uMTBrd216YX kLmNvbS94bWwvZmlsaW5nLnht bD9yZXBvPXRlbmsmaXBhZ2U9NDMxNDgxMiZkb2 M9MSZudW09MTI

2 Merck. Vioxx (rofecoxib): frequently asked questions. www.vioxx.com/rofecoxib/vioxx/consumer/faq.jsp

3 Morrison BW. Memo to Alan Nies, Barry Gertz, Beth Seidenberg. Letter to Ken Lasseter; manuscript draft for protocol 023. 18 Feb, 1998. Merck. Bates Nos MRKcom/Documents/Krumholz_Vioxx/Morrison1998.pdi

4 Food and Drug Administration, Division of AntiInflammatory, Analgesic, and Ophthalmic Drug Products. HFD-550, medical officer review. Vioxx (rofecoxib), NDA 21-042/052. Washington, DC: FDA, 1999.

5 Food and Drug Administration. FDA advisory committee briefing document NDA 21-042, s007: VIOXX gastrointestinal safety. Washington, DC: 2001.

6 Merck. Vioxx. In response to your questions: cardiovascular system, clinical profile in osteoarthritis studies. Whitehouse Station NJ: Merck, 2000. Www. vioxxdocuments.com/Documents/Krumholz_Vioxx/ Merck2000CVcard.pdi NJ0017794 to MRK-NJ0017822. www.vioxxdocuments
7 Waxman HA. Merck documents show aggressive marketing of Vioxx after studies indicated risk. US House of Representatives Committee on Government Reform, 2005. www.democrats.reform.house.gov/story.asp?ID $=848$ \& Issue $=$ Prescription+Drugs

8 Waxman HA. Memo re: the marketing of Vioxx to physicians. Washington, DC: US House of Representatives, 2005.

9 Weinblatt M. Memo to Drs. Bjorkman, Neaton, Shapiro, Silman, and Sturrock re: Interim non-endpoint safety analysis of VIGOR-unblinded minutes. November 18, 1999. Found in SNDA S-007: P088C: Appendix 3.9.2 at p.2939-2946 (Bates No. MRK-00420015464MRK-00420015471. www.vioxxdocuments.com/ Documents/Krumholz_Vioxx/VigorDSMB.pdi

10 Merck. Medical affairs procedures and policies. Procedure 23: collaborative research efforts and megatrials. Appendix 2: Merck guidelines for data and safety monitoring boards. 29 Feb, 1999. Bates Nos MRK-AFK0047772 to AFK0047791. www. vioxxdocuments.com/Documents/Krumholz Vioxx/ Merck1999guidelines.pdf

11 Reicin AS. Letter re: financial disclosure for Merck and $\mathrm{Co}$, Inc sponsored protocol entitled: "A doubleblind, randomized, stratified, parallel-group study to assess the incidence of PUBs during chronic treatment with MK-0966 or naproxen in patients with rheumatoid arthritis (VIGOR)." 4 Feb, 2000. Merck. Bates Nos MRK-MEW00012 to MRK-MEW00014. WWW. vioxxdocuments.com/Documents/Krumholz Vioxx/ ReicinWeinblatt2000.pdi.

12 Merck and Co. Multidisciplinary strategic advisory board for cox- 2 inhibitors, consulting agreement. February 29, 2000. Bates Nos MRK-STI0037747 to STI0037751. www.vioxxdocuments.com/Documents Krumholz_Vioxx/WeinblattContract.pdf.

13 Scolnick EM. Email communication to Deborah Shapiro, Alise Reicin, and Alan Nies re: Vigor. 9 Mar, 2000. Bates No MRK-ABH0016219. www.vioxxdocuments.com Documents/Krumholz Vioxx/Scolnick2000.pd

14 Drazen JM. Hidden data counfounds medical journal editors. Wall Street Journal 2006 May 19:A11.

15 Cannon CP, Curtis SP, FitzGerald GA, Krum H, Kaur A, Bolognese J, et al. Cardiovascular outcomes with etoricoxib and diclofenac in patients with osteoarthritis and rheumatoid arthritis in the multinational etoricoxib and diclofenac arthritis long-term (MEDAL) programme: a randomised comparison. Lancet 2006;368:1771-81.

16 Fries JF. Letter to Raymond Gilmartin re: physician intimidation. 9 Jan, 2001. Merck. Bates No MRK-ABH0002204 to MRK-ABH0002207. Www. vioxxdocuments.com/Documents/Krumholz_Vioxx Fries2001.pdi

17 Morrison BW. Email communication to Rhonda Sperling, Alise Reicin, Deborah Shapiro, et al. re: fw: for review [peer]: 2001-ms-2470 (full paper) - due date Monday, 27 August 2001.17 Aug, 2001. Bates Nos MRK-ACF0005697 to MRK-ACF0005699. www. vioxxdocuments.com/Documents/Krumholz_Vioxx/ Morrison2001.pdf

18 Meier B, Kolata G, Pollack A. Medicine fueled by marketing intensified trouble for pain pills. New York Times 2004 Dec 19:A1.

19 Meier B, Saul S. Marketing of Vioxx: how Merck played game of catch-up. New York Times 2005 Feb 11:A1.

20 Berenson A, Harris G, Meier B, Pollack A. Dangerous data-retracing a medical trail. New York Times 2004 Nov 14:A1.

21 IMS Health. National prescription audit plus time period 1999 to September 2004, extracted 2004. Plymouth Meeting, PA: IMS Health, 2004.

22 Targum SL. Review of cardiovascular safety database, rofecoxib. NDA 21-042, S-007. Washington, DC: FDA, 2001.www.fda.gov/ohrms/dockets/ac/01/briefing] 3677b2 06 cardio.pdi

References w1-w37 are on bmj.com 